\title{
Min-max optimization and the radial approach to the public service system design with generalized utility
}

\author{
Jaroslav Janáček ${ }^{1, *}$ and Marek Kvet $^{2}$ \\ ${ }^{1}$ Faculty of Management Science and Informatics, University of Žilina \\ Univerzitná 8215/1, 01026 Žilina, Slovakia \\ E-Mail: 〈jaroslav.janacek@fri.uniza.sk〉 \\ ${ }^{2}$ University Science Park, University of Žilina \\ Univerzitná 8215/1, 01026 Žilina, Slovakia \\ E-mail: 〈marek.kvet@uvp.uniza.sk〉
}

\begin{abstract}
The paper deals with the min-max public service system design, where the generalized utility is considered. In contrast to the formulations presented in the literature, the generalized utility defined for a public service system assumes that the user's utility comes generally from more than one located service center and the individual contributions from relevant centers are weighted by reduction coefficients depending on a center order. Given that commercial IP-solvers often fail due to enormous computational times or extreme memory demands when resolving this issue, we suggested and compared several approaches based on a bisection process with the purpose of developing an effective max-min approach to the public service system design with a generalized utility.
\end{abstract}

Key words: public service system, generalized disutility, min-max approach

Received: September 29, 2014; accepted: March 17, 2016; available online: March 31, 2016

DOI: $10.17535 /$ crorr. 2016.0005

\section{Introduction}

The design of almost any public service system $[3,5,9,11]$ includes determining center locations, from which the associated service is distributed to all users of the system. Thus the public service system structure is formed by the deployment of a limited number of service centers in a finite set of possible locations and the objective in the standard formulation is to minimize some form of disutility, which is proportional to the distance between serviced objects and the nearest service centers. This assumption of being serviced by the nearest center is not fully true, when a rescue service system is designed for random

${ }^{*}$ Corresponding author. 
service demand and limited capacity at the service centers. During momentary demand for a service, another user may occupy the nearest service center. In such situations, momentary demand is usually serviced from the second or even the third nearest center, if the second nearest center is also occupied. This randomly restricted capacity of a service center can be generalized so that the $r$ nearest centers influence the disutility perceived by a user, where $r$ is a parameter of the generalized disutility model. In this paper, the generalized disutility perceived by a user is modelled by a sum of weighted disutility contributions from the $r$ nearest centers. The weights will depend only on the order of distances from the user to the $r$ nearest centers. The $k$-th weight can be proportional to the probability that the $k-1$ nearest centers are occupied and the $k$-th nearest center is available [15]. In contrast to the min-sum public service system design, when average user disutility is to be minimized, we focus on the fair-optimal system design.

The fairness in general emerges whenever limited resources are to be fairly distributed among participants $[2,12,13]$. The strongest scheme is the so called lexicographic min-max criterion. By applying this scheme, the disutility perceived by the worst situated user is minimized first, and then disutility of the second worst situated user is minimized, unless the previously achieved disutility of the worst situated users is worsened. This approach is applied step by step to the remaining users [14]. The effective use of the approach is based on partitioning the range of all possible disutility values, which can be perceived by a user. The initial phase of the process is called the min-max optimal public service system design. In this paper, we focus on a problem-solving method for the initial phase, when the generalized disutility is considered. Based on our experiences in designing the min-sum optimal public service system, we found that the radial formulation of the problem can considerably accelerate the associated problem-solving process $[1,4,6,8]$. Furthermore, we can start from our previous research $[7,10]$, where we developed and successfully tested radial formulation of the min-sum service system design problem with the generalized disutility. We want to ascertain whether the radial approach contributes considerably to more effective problem-solving of the min-max optimal public service system design. The remainder of the paper is organized as follows. Section 2 introduces the generalized model of an individual user's disutility by considering more than one contributing center and provides a mathematical formulation of the problem based on location-allocation and radial formulations. A possible reduction of the set of relevant disutility values is also discussed. Section 3 contains a description of suggested approaches to the min-max problem. Next, Section 4 presents the numerical experiments, a comparison of the suggested approaches and finally, Sections 5 draws the final conclusions. 


\section{Generalized disutility and min-max criterion in the public service system}

\subsection{Location-allocation formulation}

The generalized model of the public service system disutility for an individual user is based on the summation of weighted disutility contributions from a given number of located service centers. The nonnegative disutility contribution $d_{i j}$ from a given service center $i$ to disutility perceived by a user located at a location $j$ grows with increasing distance between the center and user locations. Let us introduce the mapping $\Phi: R^{r} \rightarrow R^{r}$ such that a vector $\left[u_{1}, u_{2}, \ldots, u_{r}\right]$ is mapped to the vector $\left[u_{\tau(1)}, u_{\tau(2)}, \ldots, u_{\tau(r)}\right]$ using the permutation $\tau$, where the values do not decrease $u_{\tau(1)} \leq u_{\tau(2)} \ldots . \leq u_{\tau(r)}$. Based on this notation, the symbol $\Phi_{k}\left(u_{i}: i=1, \ldots, r\right)$ denotes the $k$-th component of the resulting $r$-tuple. If $I_{1}$ denotes the set of all located service centers in the public service system and $d_{i j}$ denotes the disutility contribution from service center location $i$ to the customer $j$, then the disutility of the system for the user $j$ can be expressed by (1), where $r$ denotes the given number of service centers, which take part on the utility for the user. The coefficients $q_{k}$ for $k=1 \ldots r$ are positive real values, which fulfil the following inequalities $q_{1} \geq q_{2} \geq \ldots \geq q_{r}$. According to [15], the coefficients can be proportional to the probabilities that only the $k$-th nearest center is available.

$$
\sum_{k=1}^{r} q_{k} \Phi_{k}\left(d_{i j}: i \in I_{1}\right)
$$

The problem behind the min-max optimal public service system design with generalized disutility for users is determining the service centers by minimizing perceived disutility and restricting the total number of located centers up to a given number $p$. To describe the problem, we denote $J$ as the set of user locations and $I$ as the set of possible center locations. The basic decisions in any problem-solving process relates to the location of centers for possible locations from the set $I$. These decisions will be modelled by binary variables $y_{i}$ for $i \in I$, where $y_{i}$ takes the value of 1 if a center is to be located at the location $i$, and takes the value of 0 otherwise. Further, we introduce binary variables $x_{i j k}$ for $i \in I, j \in J, k=1 \ldots r$. The variable $x_{i j k}$ takes the value of 1 specifically for the case, when user $j$ obtains the $k$-th smallest disutility contribution from the service location $i$. The associated model can be written according to [15] as follows: 
Subject to

$$
\sum_{i \in I} y_{i} \leq p
$$

$$
\begin{gathered}
x_{i j k} \leq y_{i} \quad \text { for } j \in J, i \in I, k=1, \ldots, r \\
\sum_{i \in I} x_{i j k}=1 \quad \text { for } j \in J, k=1, \ldots, r \\
\sum_{k=1}^{r} x_{i j k} \leq 1 \quad \text { for } j \in J, i \in I \\
\sum_{k=1}^{r} q_{k} \sum_{i \in I} d_{i j} x_{i j k} \leq h \text { for } j \in J \\
y_{i} \in\{0,1\} \text { for } i \in I \\
x_{i j k} \in\{0,1\} \quad \text { for } j \in J, i \in I, k=1, \ldots, r \\
h \geq 0
\end{gathered}
$$

Constraint (3) limits the number of located service centers whereas constraint (4) link up the allocation variables $x_{i j k}$ and the location variables $y_{i}$, preventing the variable $x_{i j k}$ from assigning a place $i$ without a service center to the user $j$. Constraints (5) ensure that exactly one contribution $d_{i j}$ will be assigned to the user $j$ as the $k$-th smallest contribution. Constraints (6) ensure that the contribution $d_{i j}$ will be assigned to the given user $j$ at most once. The link-up constraints (7) ensure that each perceived disutility is less than or equal to the upper bound $h$.

\subsection{Radial formulation}

We assume that the range of disutility contribution value covers only non-

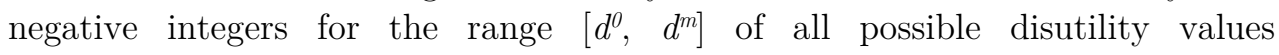
$d^{0}<d^{1}<\ldots<d^{m}$ from the matrix $\left\{d_{i j}\right\}$. The values partition the range into $m=$ $v+1$ intervals. The interval $s$ has the form $\left(d^{s}, d^{s+1}\right.$. The length of the $s$-th interval is denoted by $e_{s}$ for $s=0 \ldots v$. To describe the homogeneous system of radii determined by the values $d^{0}<d^{1}<\ldots<d^{m}$ for individual users' locations, a system of binary constants is defined so that the constant $a_{i j}{ }^{s}$ is equal to 1 if and only if the disutility contribution $d_{i j}$ for a user from location $j$ from the possible center location $i$ is less than or equal to $d_{s}$, otherwise $a_{i j}{ }^{s}$ is equal to 0 . Let the location variable $y_{i}$ have the same meaning as above. Further, we introduce auxiliary binary variables $x_{j s k}$ for $j \in J, s=0 \ldots v, k=1 \ldots r$ in order to model the disutility contribution value of the $k$-th nearest service center to the user $j$. The variable $x_{j s k}$ takes the value 1 if the $k$-th smallest disutility contribution for the 
customer $j \in J$ is greater than $d_{s}$ and it takes the value 0 otherwise. Then the expression $e_{0} x_{j 0 k}+e_{1} x_{j 1 k}+e_{2} x_{j 2 k}+e_{3} x_{j 3 k}+\ldots+e_{v} x_{j v k}$ constitutes the $k$-th smallest disutility contribution $d_{j^{*}}^{k}$ for customer $j$. Under above-mentioned preconditions, we can describe the min-max-optimal public service system design problem (11) - (17) using the variables and other notations introduced above.

\section{Minimize $h$}

Subject to

$$
\sum_{i \in I} y_{i} \leq p
$$

$$
\begin{gathered}
\sum_{k=1}^{r} x_{j s k}+\sum_{i \in I} a_{i j}^{s} y_{i} \geq r \text { for } j \in J, s=0, \ldots, v \\
\sum_{k=1}^{r} q_{k} \sum_{s=0}^{v} e_{s} x_{j s k} \leq h \text { for } j \in J \\
y_{i} \in\{0,1\} \text { for } i \in I \\
x_{j s k} \in\{0,1\} \text { for } j \in J, s=0, \ldots, v, k=1, \ldots, r \\
h \geq 0
\end{gathered}
$$

Constraint (12) defines an upper bound $p$ on the number of located centers. Since the second term of the left-hand-side of (13) gives the number of centers located within radius $d^{s}$ from the user location $j$, constraint (13) ensures for a given $j$ that the sum of variables $x_{j s k}$ over $k=1 \ldots r$ expresses the complement of that number for the value $r$. The link-up constraints (14) ensure that each perceived disutility is less than or equal to the upper bound $h$. Validity of the assertion that the expression on the left-hand side of (14) expresses the sum $q_{1} d_{i 1, j}+q_{2} d_{i 2, j}+\ldots+q_{r} d_{i r, j}$ of weighted relevant disutility values from the $r$ nearest service centers $i 1, i 2, \ldots$, ir to $j$, is based on the following reasoning. It can be easily found that the minimal sum of the variables $x_{j s k}$ over $k=1 \ldots r$ completes the number of located service centers in the radius $s$ from user location $j$ to the number $r$. In this way, the sum gives the number $t$ of the nearest service centers, whose disutility contribution is greater than or equal to the value $d_{s}$. As the sequence of $q_{k}$ decreases, only $x_{j s k}$ for $k=r-t+1, r-t+2 \ldots r$ must be equal to one for the given $j$ and $s$. This implies that the biggest disutility contribution is assigned the smallest value of $q_{k}$. The left-hand-side of (14) is pushed down by the optimization process, and subsequently the constraints $x_{j s k} \leq x_{j s-1, k}$ for $s=1 \ldots$ $v$ must hold due to the construction of $a_{i j}{ }^{s}$ and constraints (13) and furthermore, the constraints $x_{j s k} \leq x_{j s k+1}$ for $k=1 \ldots r$ - 1 must hold due to convexity given by a decreasing sequence of $q_{k}$. 


\subsection{Discussion on the location-allocation and radial approaches}

Both the above mentioned approaches were broadly tested and compared. The studies were performed for the case of min-sum optimization, which is used when designing the min-sum optimal public service system. In that case, the sum of all disutility values perceived by all users is minimized. That means that the link-up constraints (7) and (14) were absent in models (2)-(10) and (11)(17), respectively. It was found $[4,10]$ that the radial approach considerably (in orders) outperformed the location-allocation approach in terms of computational time when designing the min-sum optimal public service system. Nevertheless, our preliminary experiments suggested that the link-up constraints for the upper bound definition significantly spoil the convergence of the computational process based on the branch and bound principle. This deterioration was so strong that it discharged the better convergence characteristic of the radial approach. We tried to improve convergence of the location-allocation and radial approaches using a trick and which was used in fuzzy optimization to avoid solving a nonlinear problem. This approach consists in fixing the upper bound $h$ at some chosen value and solving the problem with some surrogate objective function to find whether the original problem has a feasible solution with the fixed objective function value. This process is repeated for decreasing values of $h$ until no feasible solution exists.

Furthermore, the generalized disutility concept is accompanied by another disadvantage comparing to the classical disutility coming only from the nearest located center. When designing the min-max public service system, and only a classical disutility model. Then, the minimal disutility value of the worst situated user can be used as a threshold and all disutility values exceeding the threshold can be excluded from the process. The general disutility model does not have this useful property, as we will show in the next sub-section.

\subsection{Impossibility of reducing general disutility}

The perceived disutility model is based on the matrix $\left\{d_{i j}\right\}$ of integer contributions from a possible center location $i$ to a user located at a place $j$. The set of all values contained in the matrix $\left\{d_{i j}\right\}$ can be represented by an ordered sequence of unique values $d_{0}<d_{1}<d_{2}<\ldots d_{m}$. In such cases, when the classical disutility model is considered, i.e. $r=1$, and a solution of the problems (2) (10) or $(11)-(17)$ is found for $h=d_{t}$ at step $t$ of the bisection process, then both problems can be considerably reduced. The problem (2) - (10) can be reduced by excluding all the allocation variables, associated with disutility value $d_{i j}>d_{t}$. The problem $(11)-(17)$ can be reduced in the parameter $v$ so that $v$ can be set at the value $d_{t}-1$ instead of the original value $d_{m}-1$. This reduction can be used in further steps, even if only the initial phase of the lexicographic 
minimization or the complete process is continued. We will show in the next example that this straightforward reduction is impossible, when using the generalized utility with $r \geq 2$.

Let us consider the network graph depicted in Figure 1. The graph consists of the vertex set $V=\{1,2, \ldots, 6, j, j\}$ and the set of weighted edges, where the lengths of the edges are placed at individual edges as depicted in the figure.

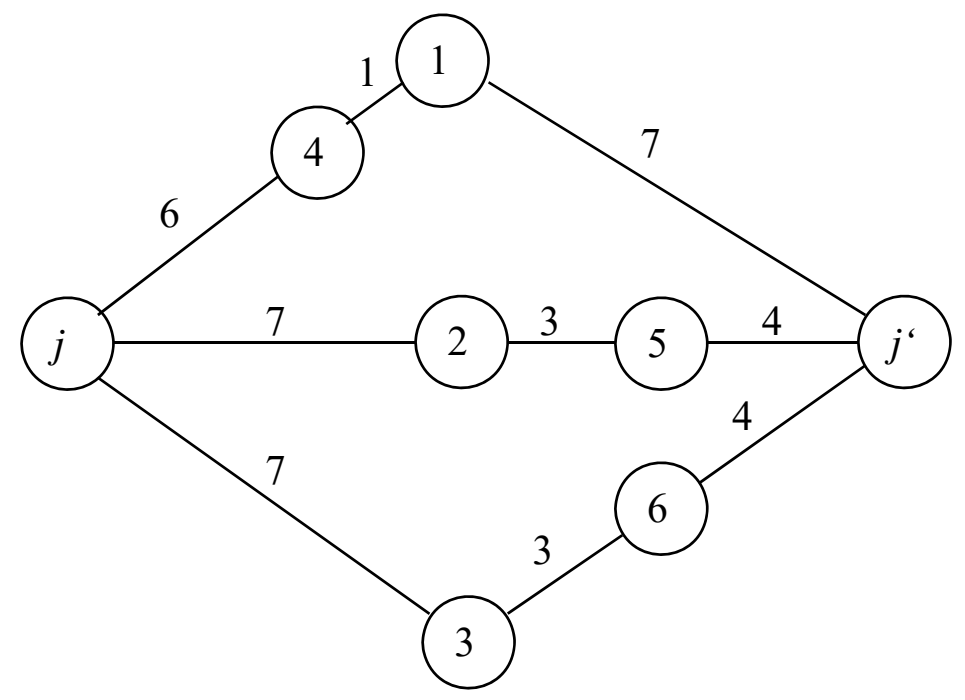

Figure 1: Example network

The counter example can be defined on the network, where $J=\{j, j$ ' $\}$ represents the set of user locations and $I=\left\{\begin{array}{llll}1,2 \ldots & 6\end{array}\right\}$ is the set of possible center locations. The matrix $\left\{d_{i j}\right\}$ of the potential disutility contributions from the center locations is presented in Table 1.

\begin{tabular}{|c|c|c|}
\hline Center locations & $d_{i j}$ & $d_{i j^{\prime}}$ \\
\hline 1 & 7 & 7 \\
\hline 2 & 7 & 7 \\
\hline 3 & 7 & 7 \\
\hline 4 & 6 & 8 \\
\hline 5 & 10 & 4 \\
\hline 6 & 10 & 4 \\
\hline
\end{tabular}

Table 1: Potential disutility contributions from the center locations to the user locations $j$ and $j$.

The disutility contributions from individual centers to a user are defined here as the lengths of the shortest paths from the user location to the center 
locations. We consider the counterexample parameters $r=3$ and $p=3$. The reduction coefficients $q_{k}$ for $k=1,2,3$ are $1,0.2$ and 0.1 , respectively. The objective is to find three locations from $I$ so that the maximal value from generalized disutility perceived by users $j$, and $j$ ' is minimal. It can be easy found that three centers located at locations from $I_{1}=\{1,2,3\}$ represent a feasible solution, where the generalized disutility value for users $j$ and $j$ ' are $q_{1} d_{1 j}+\quad q_{2} d_{2 j}+\quad q_{3} d_{3 j} \quad=1^{*} 7+0.2^{*} 7+0.1^{*} 7=9.1 \quad$ and $\quad q_{1} d_{1 j^{*}}+\quad q_{2} d_{2 j^{*}}+\quad q_{3} d_{3 j^{*}=}$ $1^{*} 7+0.2^{*} 7+0.1 * 7=9.1$, respectively. Since the complete sequence of the disutility contribution values consists of the values $4,6,7,8$ and 10 , and the two highest values are not used in the discussed solution, it may seem that the values 8 and 10 could be excluded from the next steps of the computational process, similarly to the case of $r=1$. However, in contrast to the classical case, the reduction of these values in the presented example excludes the better solution given by $I_{1}=$ $\{4,5,6\}$, where generalized disutility values for $j$ and $j$ ' are $q_{1} d_{4 j}+q_{2} d_{5 j}+q_{3} d_{6 j}$ $=1 * 6+0.2 * 10+0.1 * 10=9 \quad$ and $\quad q_{1} d_{4 j^{*}}+\quad q_{2} d_{5 j^{*}}+\quad q_{3} d_{6 j}=1 * 4+0.2 * 4+0.1 * 8=5.6$, respectively. The maximum of these general disutility values for $j$ and $j$ ' is 9 , but this solution would be unattainable if the disutility values 8 and 10 were excluded.

\section{Min-max problem solving methods}

We have to note that the min-max problem solution can be obtained directly by using a common IP-solver to solve the problems described by models (2)-(10) or (11)-(17).

Another more promising approach is based on a bisection search covering the range of the generalized disutility values. This search tries to find the minimal value $G^{*}$ for a feasible service system design, where no user perceives a disutility higher than $G^{*}$.

The searching process consists of individual steps, where step $t$ answers the question as to whether there is a feasible solution with maximal perceived disutility less than or equal to a given value $G_{t}$ determined at step $t$ of the bisection process.

If the location-allocation formulation of the public service system design problem is considered and a common IP-solver is used, then there are two different formulations of the particular problem solved at step $t$. The first formulation consists in minimizing expression (18) subject to (3)-(6), (8), (9) and (19).

$$
\sum_{j \in J} \sum_{k=1}^{r} q_{k} \sum_{i \in I} d_{i j} x_{i j k}
$$




$$
\sum_{k=1}^{r} q_{k} \sum_{i \in I} d_{i j} x_{i j k} \leq G_{t} \text { for } j \in J
$$

If the optimization process of the used IP-solver leads to an optimal solution for the above problem, a feasible solution exists and the lower value $G_{t+1}$ can be tested. In the opposite case, the searched value $G^{*}$ is higher than $G_{t}$.

In the second formulation, the auxiliary variables $h_{j} \geq 0$ for $j \in J$ are introduced and the minimization problem (20) subject to (3)-(6), (8), (9), (21) and (22) is solved.

$$
\begin{gathered}
\sum_{j \in J} h_{j} \\
\sum_{k=1}^{r} q_{k} \sum_{i \in I} d_{s} x_{i j k} \leq G_{t}+h_{j} \text { for } j \in J \\
h_{j} \geq 0 \text { for } j \in J
\end{gathered}
$$

If the optimization process of the used IP-solver leads to an optimal solution of the above problem and the optimal objective function value is zero, a feasible solution for the $t$-th step problem exists and the lower value $G_{t+1}$ can be tested. In the opposite case, the searched value $G^{*}$ is higher than $G_{t}$.

A similar construction can be developed for radial formulation, where the first formulation consists of minimizing expression (23) subject to (12), (13), (15), (16) and (24).

$$
\begin{gathered}
\sum_{j \in J} \sum_{k=1}^{r} q_{k} \sum_{s=0}^{v} e_{s} x_{j s k} \\
\sum_{k=1}^{r} q_{k} \sum_{s=0}^{v} e_{s} x_{j s k} \leq G_{t} \text { for } j \in J
\end{gathered}
$$

In the second formulation, the auxiliary variables $h_{j} \geq 0$ for $j \in J$ are also introduced and the minimization problem (25) subject to (12), (13), (15), (16), (26) and (22) is solved.

$$
\begin{gathered}
\sum_{j \in J} h_{j} \\
\sum_{k=1}^{r} q_{k} \sum_{s=0}^{v} e_{s} x_{j s k} \leq G_{t}+h_{j} \text { for } j \in J
\end{gathered}
$$


Further, the zero value of the objective function value indicates that a feasible solution for the $t$-th step problem exists.

\section{Computational study}

To compare the four approaches mentioned in the Section 3 and the two approaches in the Section 2, several initial experiments were performed. The benchmarks were obtained by modifying a model of a real emergency health care system, which was originally designed for a self-governing region of Žilina. This system covers the demands of 315 communities - towns and villages, over a region covered by 36 ambulance vehicles, where each represents a service center. These communities were considered as elements of the set $J$ of users' locations and as elements of the set $I$ of possible service center locations. The disutility contribution from a possible location $i$ to a user location $j$ was represented by the road network distance between the two locations. In the benchmarks, the generalized disutility perceived by a user sharing a given location was the sum of three distances from the user's location to the three nearest vehicle locations. The distances are multiplied by the reduction coefficients so that the largest coefficient multiplies the smallest distance etc. The four triples $\boldsymbol{q}^{1}, \boldsymbol{q}^{2}, \boldsymbol{q}^{3}, \boldsymbol{q}^{4}$ of the reduction coefficients define the individual benchmarks, and the symbols of the triples are used for distinguishing the results obtained using individual approaches on the benchmarks. The used triples were $\boldsymbol{q}^{1}=[1,0.2,0.1], \boldsymbol{q}^{2}=[1$, $0.1,0.05], \boldsymbol{q}^{3}=[1,0.5,0.2]$ and $\boldsymbol{q}^{4}=[1,0.8,0.5]$.

The tested approaches are called LA_EX, RA_EX, LA_BG, RA_BG, LA_BGh and RA_BGh. The prefixes LA and RA denote location-allocation and radial formulation, respectively. The suffix EX denotes the exact approach, when IP-solver solves the problem described by program (2)-(10) or (11)-(17). The suffixes BG and BGh denote bisection approaches, where the IP-solver solves the optimization problem for a fixed value $G_{t}$ at each step $t$. The denotation BG corresponds to the models (18), (3)-(6), (8), (9), (19) or (23), (12), (13), (15), (16), (24), while the denotation BGh is used for the models (20), (3)-(6), (8), (9), (21), (22) or (25), (12), (13), (15), (16), (26), (22) depending on location-allocation or radial formulations. To solve the problems described by the mentioned models, optimization software FICO Xpress 7.3 (64bit, release 2012) was used and the experiments were run on a PC equipped with the Intel® Core ${ }^{\mathrm{TM}}$ i7 3610 QM processor, at $2.3 \mathrm{GHz}$ and with $8 \mathrm{~GB}$ RAM. The preliminary experiments showed that the IP-solver required unpredictable computational time. When the middle-size integer programming problem is solved to optimality, we decided to test each approach during a one-hour period. The LA_EX and RA_EX approaches were run for an hour to solve the problem for each triple of the reduction coefficients and the objective function values of the best found feasible solutions are presented in Table2. The best found 
objective function values in the computation study were put in the row denoted as "Best found solution".

\begin{tabular}{|l|c|c|c|c|}
\hline \multicolumn{1}{|c|}{ Approaches } & $q^{1}$ & $q^{2}$ & $q^{3}$ & $q^{4}$ \\
\hline LA_EX & 100.4 & 96.3 & 77.2 & 87.5 \\
\hline RA_EX & 25.2 & 18.95 & 32.2 & 43.5 \\
\hline Best found solution & 20.8 & 17.25 & 30.6 & 43.5 \\
\hline
\end{tabular}

Table 2: The best found objective function values reached by the $L A \cdot E X$ and $R A \cdot E X$ approaches over a one-hour period.

Comparison of the approaches are based on bisection met with several technical obstacles due to the fact that optimization procedures of the IP-solver are run at each step of the bisection process, and furthermore, only the first feasible solution was searched in order to complete the step, when LA_BG and RA_BG are tested. Since the bisection process needs at most seven steps to finish the optimization process, the performance of one step was limited to 450 seconds, to prevent the solver from doing a long search for verifying unnecessary optimality. On the other hand, this restriction may cause premature stopping of the particular search before the first feasible solution is found, even if a feasible solution exists. The bisection process may then fail in separating the correct interval containing the searched minimal value. That is why two parameters of the bisection processes are studied. The first parameter is the computation time $\mathrm{CT}$ in seconds and the second one is the number CS of steps, which do not terminate prematurely. The symbol $G^{*}$ denotes the best found value of the generalized disutility, which corresponds to the maximal disutility perceived by the most exposed users of the designed public service system. The results of experiments are presented in Table 3.

\begin{tabular}{|c|c|c|c|c|c|c|c|c|c|c|c|c|}
\hline & & $q^{1}$ & & & $q^{2}$ & & & $q^{3}$ & & & $q^{4}$ & \\
\hline Approaches & $\mathrm{CS}$ & $\mathrm{CT}[\mathrm{s}]$ & $G^{*}$ & $\mathrm{CS}$ & $\mathrm{CT}[\mathrm{s}]$ & $G^{*}$ & $\mathrm{CS}$ & $\mathrm{CT}[\mathrm{s}]$ & $G^{*}$ & $\mathrm{CS}$ & $\mathrm{CT}[\mathrm{s}]$ & $G^{*}$ \\
\hline LA_BG & 7 & 1535 & 20.8 & 7 & 1030 & 17.3 & 7 & 1883 & 30.6 & 6 & 1237 & 46.0 \\
\hline LA_BGh & 5 & 2548 & 53.3 & 3 & 2922 & 40.3 & 5 & 2704 & 48.8 & 4 & 2587 & 59.8 \\
\hline RA_BG & 7 & 2186 & 20.8 & 6 & 1709 & 23.0 & 6 & 2211 & 34.0 & 6 & 1999 & 46.0 \\
\hline RA_BGh & 5 & 2640 & 24.7 & 6 & 1687 & 23.0 & 5 & 1779 & 35.7 & 4 & 1933 & 52.9 \\
\hline
\end{tabular}

Table 3: Comparison of the bisection approaches

As can be seen, the bisection approaches were able to obtain a better solution than the exact approaches in the limited time, but they were not too reliable as concerns the possibility to fail at the particular steps of the bisection process. The approaches BG (meaning LA_BG and RA_BG) perform better than the BGh approach. Surprisingly, the LA_BG approach outperformed the radial formulation approach. The unreliability of the bisection approaches 
evoked the idea to start the bisection process with an initial pre-search based on strengthening of the constraints (24). We replaced constraints (24) with (27).

$$
\sum_{s=0}^{v} e_{s} x_{j s k} \leq G_{t} / \sum_{t=1}^{r} q_{t}=\sum_{s=0}^{f-1} e_{s} \quad \text { for } j \in J, k=1, \ldots, r
$$

This approximation of the original constraints enabled solving the problem (25), (28), (30), (12) and (15) instead of (23), (12), (13), (15), (16) and (24) at each step of the bisection process.

$$
h_{j}+\sum_{i \in I} a_{i j}^{f} y_{i} \geq r \quad \text { for } j \in J
$$

This initial phase gives the result presented in Table 4 .

\begin{tabular}{|c|c|c|c|c|c|c|c|}
\hline $\boldsymbol{q}^{1}$ & & $\boldsymbol{q}^{2}$ & & $\boldsymbol{q}^{3}$ & & $\boldsymbol{q}^{4}$ & \\
\hline $\mathrm{CT}[\mathrm{s}]$ & $G^{*}$ & $\mathrm{CT}[\mathrm{s}]$ & $G^{*}$ & $\mathrm{CT}[\mathrm{s}]$ & $G^{*}$ & $\mathrm{CT}[\mathrm{s}]$ & $G^{*}$ \\
\hline 0.39 & 32.5 & 0.38 & 28.8 & 0.39 & 42.5 & 0.36 & 57.5 \\
\hline
\end{tabular}

Table 4: Results of the initial phase

\section{Conclusions}

This paper suggests and compares solving techniques for the min-max public service system design with the generalized disutility model. The generalized disutility model impacts the complexity of the problems, where such problems are resolved using the presented techniques. The consequence of using the generalized disutility model when designing the min-max optimal public service system is that the suitability of the common approaches to the min-sum public service system design changes considerably. We suggested several bisection approaches to the problem and explored their effectiveness. In contrast to previously obtained results for min-sum designs, the radial approach seems not to outperform the location-allocation approach. Furthermore, we suggested improving the bisection process requiring the insertion of so a called initial phase, which in turn provides a good starting solution with good objective function value in very short time. This initial phase algorithm is much faster than the continuing bisection process. The min-max design, treated and solved in this paper, plays not only important role in lexicographic optimization, but also considerably reduces the set of effective general disutility values.

\section{Acknowledgement}

This work was supported by the research grants VEGA 1/0518/15 "Resilient rescue systems with uncertain accessibility of service", VEGA 1/0463/16 "Economically efficient charging infrastructure deployment for electric vehicles in smart cities and communities", APVV-0760-11 "Designing of Fair Service 
Systems on Transportation Networks" and also by the project University Science Park of the University of Žilina (ITMS: 26220220184) supported by the Research \& Development Operational Program and funded by the European Regional Development Fund.

\section{References}

[1] Avella, P., Sassano, A. and Vassil'ev, I. (2007). Computational study of large scale p-median problems. Mathematical Programming, 109, 89-114.

[2] Bertsimas, D., Farias, V. F. and Trichakis, N. (2011). The Price of Fairness. Oper. Res., 59, 17-31.

[3] Current, J., Daskin, M. and Schilling, D. (2002). Discrete network location models. In: Drezner, Z. (Ed.). Facility location - Applications and theory (pp. 81-118). Berlin: Springer.

[4] García, S., Labbé, M. and Marín, A. (2011). Solving large p-median problems with a radius formulation. INFORMS Journal on Computing, 23(4), 546-556.

[5] Ingolfsson, A., Budge, S. and Erkut, E. (2008). Optimal ambulance location with random delays and travel times. Health Care management science, 11(3), 262-274.

[6] Janáček, J. (2008). Approximate covering models of location problems. In: Lecture Notes in Management Science: Proceedings of the 1st International Conference ICAOR '08, Vol. 1, Sept. 2008, Yerevan, Armenia, (pp. 53-61).

[7] Janáček, J. (2014). Radial approach to the emergency public service system design with generalized system utility. International Journal of Applied Mathematics and Informatics, 8, 7-14

[8] Janáček, J. and Kvet, M. (2014). Relevant network distances for approximate approach to the p-median problem. In: Operations Research Proceedings 2012: Selected Papers of the International Conference of the German operations research society (GOR), September 4-7 2012, Leibniz Univesität Hannover, Germany (pp. 123-128). Springer.

[9] Jánošíková, L. (2007). Emergency medical service planning. Communications Scientific Letters of the University of Žilina, 9(2), 64-68.

[10] Kvet, M. (2014). Computational study of radial approach to public service system design with generalized utility. In: Digital Technologies 2014: Proceedings of the 10th International IEEE Conference, Žilina, (pp. 198-208).

[11] Marianov, V. and Serra, D. (2002). Location problems in the public sector. In: Drezner, Z. (Ed.). Facility location - Applications and theory (pp 119-150). Berlin: Springer.

[12] Marsh, M. and Schilling, D. (1994). Equity measurement in facility location analysis. European Journal of Operational Research, 74, 1-17.

[13] Nash, J. (1950). The bargaining problem. Econometrica, 18(2), 155-162.

[14] Ogryczak, W. and Sliwinski, T. (2006). On direct methods for lexicographic minmax optimization. In: Gavrilova M. et al. (Eds.): ICCSA 2006, LNCS 3982, (pp. 802-811). Berlin Heidelberg: Springer.

[15] Snyder, L.V. and Daskin, M. S. (2005). Reliability models for facility location: The expected failure cost case. Transport Science, 39(3), 400-416. 